\title{
The optical blocking filter for the ATHENA wide field imager: ongoing activities towards the conceptual design
}

M. Barbera, G. Branduardi-Raymont, A. Collura, A. Comastri, J. Eder, et al.

M. Barbera, G. Branduardi-Raymont, A. Collura, A. Comastri, J. Eder, T. Kamisiński, U. Lo Cicero, N. Meidinger, T. Mineo, S. Molendi, G. Parodi, A. Pilch, L. Piro, M. Rataj, G. Rauw, L. Sciortino, S. Sciortino, P. Wawer, "The optical blocking filter for the ATHENA wide field imager: ongoing activities towards the conceptual design," Proc. SPIE 9601, UV, X-Ray, and GammaRay Space Instrumentation for Astronomy XIX, 960109 (24 August 2015); doi: $10.1117 / 12.2189326$

SPIE Event: SPIE Optical Engineering + Applications, 2015, San Diego, California, United States 


\title{
The optical blocking filter for the ATHENA Wide Field Imager: ongoing activities towards the conceptual design
}

\author{
M. Barbera*a,b , G. Branduardi-Raymont ${ }^{\mathrm{c}}$, A. Collura ${ }^{\mathrm{b}}$, A. Comastri ${ }^{\mathrm{d}}$, J. Eder $^{\mathrm{e}}$, T. Kamisiński $^{\mathrm{f}}$, \\ U. Lo Cicero ${ }^{\text {b,a }}$, N. Meidinger ${ }^{\mathrm{e}}$, T. Mineo ${ }^{\mathrm{g}}$, S. Molendi ${ }^{\mathrm{h}}$, G. Parodi ${ }^{\mathrm{i}}$, A. Pilch ${ }^{\mathrm{f}}$, L. Piro $^{\mathrm{j}}$, M. Rataj ${ }^{\mathrm{k}}$, \\ G. Rauw ${ }^{1}$, L. Sciortino ${ }^{\mathrm{a}, \mathrm{b}}, \mathrm{S}$. Sciortino ${ }^{\mathrm{b}}, \mathrm{P}$. Wawer ${ }^{\mathrm{k}}$
}

${ }^{a}$ Università degli Studi di Palermo, Dipartimento di Fisica e Chimica, Via Archirafi 36, 90123

Palermo, Italy; ${ }^{b}$ Istituto Nazionale di Astrofisica, Osservatorio Astronomico di Palermo G.S. Vaiana, Piazza del Parlamento 1, 90134 Palermo, Italy; ' University College London, Department of Space and Climate Physics, Mullard Space Science Laboratory, Holmbury St Mary, Dorking, Surrey RH5 6NT, United Kingdom; ${ }^{d}$ Istituto Nazionale di Astrofisica, Osservatorio Astronomico di Bologna, Via Ranzani 1, 40127 Bologna, Italy; ${ }^{\mathrm{e}}$ Max-Planck-Institut fuer extraterrestrische Physik,

Giessenbachstrasse, 85748 Garching, Germany; ${ }^{\mathrm{f}}$ Department of Mechanics and Vibroacoustics, AGH University of Science and Technology, al. A. Mickiewicza 30, 30-059 Kraków, Poland; ${ }^{\mathrm{g}}$ Istituto Nazionale di Astrofisica, Istituto di Astrofisica Spaziale e Fisica Cosmica di Palermo, Via U. La Malfa, 153, 90146 Palermo, Italy; ${ }^{h}$ Istituto Nazionale di Astrofisica, Istituto di Astrofisica Spaziale e Fisica Cosmica di Milano, Via E. Bassini 15, 20133 Milano, Italy;

${ }^{i}$ BCV progetti s.r.l., via S. Orsola 1, 20123 Milano, Italy; ${ }^{j}$ Istituto Nazionale di Astrofisica, Istituto di Astrofisica e Planetologia Spaziali, Via Fosso del Cavaliere 100, 00133 Roma, Italy; ${ }^{\mathrm{k}}$ Space Research Center, Polish Academy of Sciences, Bartycka 18A, 00-716 Warsaw, Poland; ${ }^{1}$ University of Liège, Department of Astrophysics, Geophysics \& Oceanography, Allée du 6 Août B5c, B-4000 Liège, Belgium;

\begin{abstract}
ATHENA is the L2 mission selected by ESA to pursue the science theme "Hot and Energetic Universe" (launch scheduled in 2028). One of the key instruments of ATHENA is the Wide Field Imager (WFI) which will provide imaging in the $0.1-15 \mathrm{keV}$ band over a $40^{\prime} \times 40^{\prime}$ large field of view, together with spectrally and time-resolved photon counting. The WFI camera, based on arrays of DEPFET active pixel sensors, is also sensitive to UV/Vis photons. Optically generated electron-hole pairs may degrade the spectral resolution as well as change the energy scale by introducing a signal offset. For this reason, the use of an X-ray transparent optical blocking filter is needed to allow the observation of all type of X-ray sources that present a UV/Visible bright counterpart.

In this paper, we describe the main activities that we are carrying on for the conceptual design of the optical blocking filter, that will be mounted on the filter wheel, in order to satisfy the scientific requirements on optical load from bright $\mathrm{UV} / \mathrm{Vis}$ astrophysical source, to maximize the X-ray transmission, and to withstand the severe acoustic and vibration loads foreseen during launch.
\end{abstract}

Keywords: X-rays, Instrumentation for Astrophysics, ATHENA, WFI, filters

\section{INTRODUCTION}

ATHENA[1] is an X-ray observatory-class mission designed by a large European consortium to address the "Hot and Energetic Universe" science theme[2] selected by the European Space Agency for L2 - the second Large-class mission

*marco.barbera@unipa.it; phone+39091233613

UV, X-Ray, and Gamma-Ray Space Instrumentation for Astronomy XIX, edited by Oswald H. Siegmund Proc. of SPIE Vol. 9601, 960109 - (C) 2015 SPIE · CCC code: 0277-786X/15/\$18 - doi: 10.1117/12.2189326 
within the Cosmic Vision science program scheduled for launch in 2028. Thanks to the innovative technology of its optics[3] and the most advanced focal plane X-ray instrumentation, the ATHENA mission will deliver X-ray (0.2-12 $\mathrm{keV}$ ) wide field imaging, timing and high energy resolution spectroscopy capabilities far beyond those of any existing or approved future missions.

One of the two detectors that will alternatively operate at the focal plane of the large area telescope of ATHENA is the Wide Field Imager, which will provide imaging in the $0.1-15 \mathrm{keV}$ band over a $40^{\prime} \times 40^{\prime}$ large field of view, simultaneously with spectrally and time-resolved photon counting[4],[5].

The WFI camera, based on arrays of DEPFET active pixels, is also sensitive to UV/Vis photons. Optically generated photo-electrons may degrade the spectral resolution as well as change the energy scale by introducing a signal offset. For this reason, the use of an X-ray transparent optical blocking filter (OBF) is needed to allow the observation of X-ray sources that present UV/Visible bright counterparts. The WFI current design includes an OBF directly deposited on-chip consisting of a multilayer of $30 \mathrm{~nm} \mathrm{SiO}{ }_{2}-20 \mathrm{~nm} \mathrm{Si} \mathrm{Si}_{3}-90 \mathrm{~nm}$ Al which already provides nearly $10^{-5}$ attenuation in the UV/Vis (Figure 1).

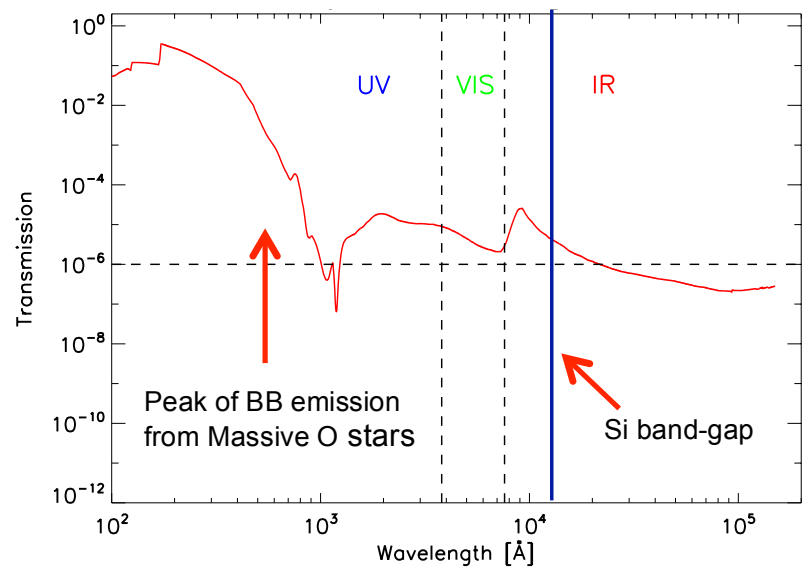

Figure 1. Calculated transmission of the multi-layer optical blocking filter directly deposited on the DEPFET.

Such attenuation factor is not enough for all astrophysical sources, for this reason an additional OBF mounted on a filter wheel $(\mathrm{FW})$ is needed to observe sources with very bright UV/Vis counterparts such as massive stars. The FW will also contain the calibration source, the closed aperture for background calibration and detector safety protection, and the free aperture (Figure 2 left panel). The filter wheel assembly (FWA) is placed in the structure of the WFI (Figure 2 right panel) and the current plan is to launch it in atmospheric pressure. For this reason, the FWA should be properly designed to reduce the severe mechanical vibration and acoustic load onto the OBF during launch.

The large size of the OBF, due to the large detector FOV and telescope aperture, the small thickness to maintain a high transparency in X-rays still providing adequate UV/Vis attenuation, the mechanical robustness to withstand launch stresses and in particular acoustic load (not present for Chandra and XMM-Newton where the filters were launched in vacuum), and the need to protect the detector from contamination (possible requirement on heating for decontamination), makes the design and construction of the OBF and FWA a big challenge.

In the following paragraphs we present the main results of the ongoing activities aimed at designing the OBF and procuring a first set of filter samples to perform optical/mechanical characterization tests which are necessary to verify the filter conceptual design and increase the TRL. 

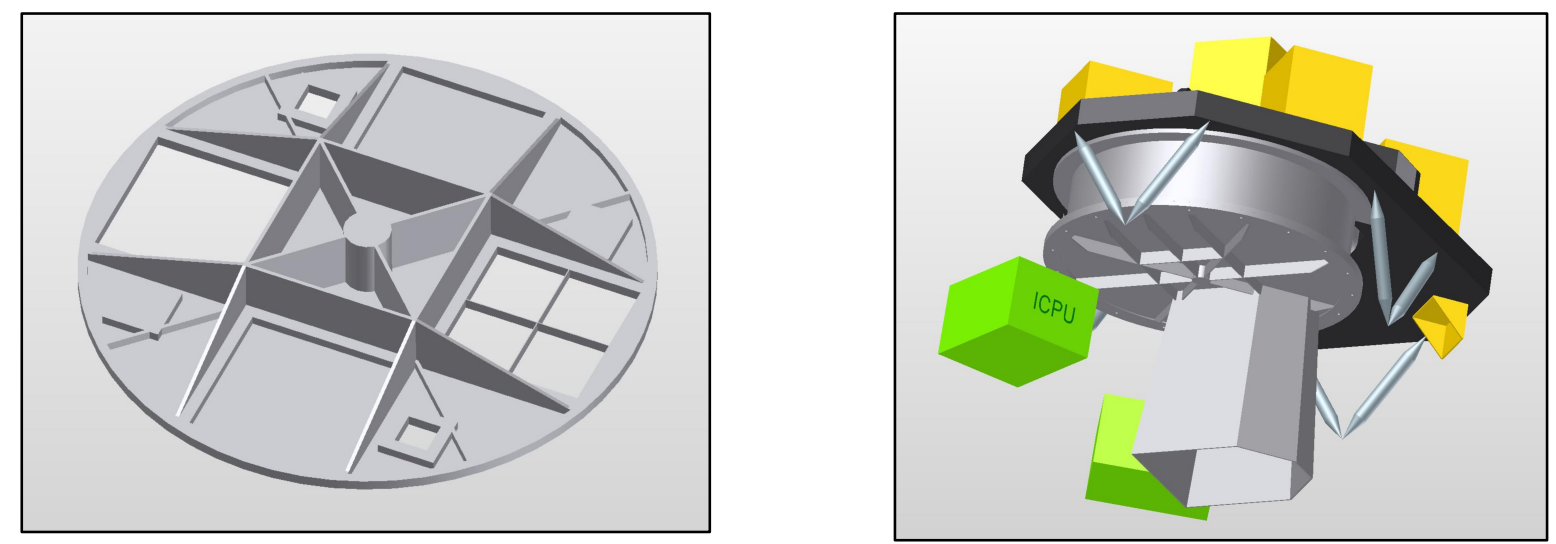

Figure 2. Left panel - Schematic drawing of the filter wheel showing the four positions: open, closed, calibration source and optical blocking filter. The diameter of the wheel is currently $\sim 60 \mathrm{~cm}$. Right panel - Schematic drawing of the filter wheel assembly and entrance baffle attached to the main structure of the WFI.

\section{FILTER BASELINE DESIGN}

Large size filters made of sub-micron thickness polyimide films coated with aluminum have been used in many recent missions such as Chandra[6][7], and XMM-Newton[8] demonstrating reliability and long-term stability. As an example, the thin filters of the EPIC camera on board XMM-Newton are nearly $75 \mathrm{~mm}$ diameter and consist of an unsupported $160 \mathrm{~nm}$ thick Polyimide film coated with an aluminum layer $40 \mathrm{~nm}$ thick. Such filters have survived launch and are still working with no clear sign of degradation[9][10]. More recently, large area $\left(80 \times 120 \mathrm{~mm}^{2}\right)$ filters consisting of a $1 \mu \mathrm{m}$ thick LUXfilm ${ }^{\circledR}$ polyimide coated with $40 \mathrm{~nm}$ of aluminum, both unsupported and supported by a stainless steel mesh with $90 \%$ open area, have been investigated for the LAD[11] experiment on LOFT[12][13] (ESA M4 candidate mission). Both filters mounted inside a cavity representing the LAD Micro-channel Plate (MPC) collimator frame and box have survived the acoustic tests with a sound pressure levels equivalent to the launch of the Soyuz vehicle[14].

Such positive experiences with polyimide films coated with aluminum have suggested us to consider these materials as the baseline for the WFI OBF. Beside its excellent mechanical properties, polyimide strongly absorbs radiation in the $\mathrm{UV}$ at wavelengths shorter than about $3500 \AA[15]$ providing the required additional attenuation in the spectral region where the black body emission of high mass stars peaks $(600-2000 \AA)$.

The definition of the thicknesses of the polyimide film and the aluminum layer will be the result of an iterative process largely driven by the scientific requirements on detectability of X-ray sources with bright UV/Vis counterparts (see next paragraph).
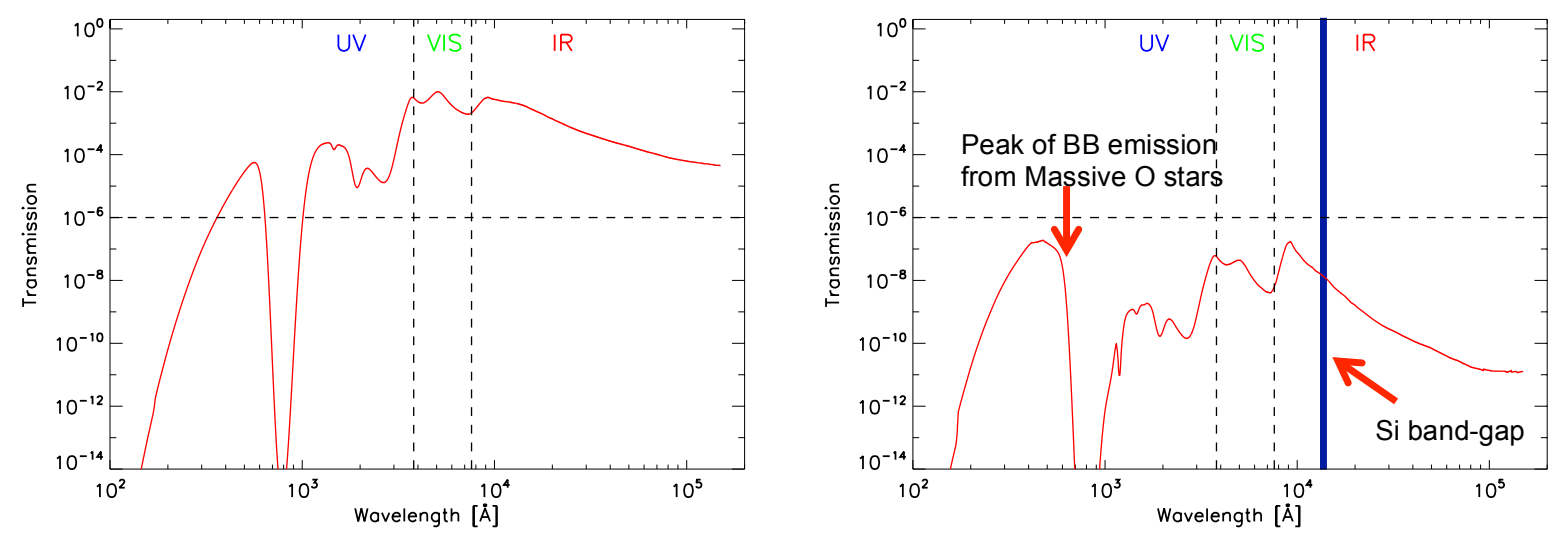

Figure 3. Left panel - Calculated transmission for an OBF mounted on the FW consisting of $200 \mathrm{~nm}$ of polyimide and $40 \mathrm{~nm}$ of aluminum. Right panel - Total transmission for the FW OBF and the OBF directly deposited on-chip. 
The left panel in figure 3 shows the UV/Vis/IR transmission calculated for an OBF design consisting of $200 \mathrm{~nm}$ of polyimide and $40 \mathrm{~nm}$ of aluminum, while the right panel shows the total transmission of such filter and the previously described OBF deposited on-chip. The calculations are derived by the use of the matrix formulation of the boundary conditions of the electromagnetic field[16], with the refractive index of aluminum derived from three different sources for the wavelength range $0.01653 \div 32 \mu \mathrm{m}[17], 33.333 \div 200 \mu \mathrm{m}$ [18], and $248 \div 1240 \mu \mathrm{m}[19]$, respectively, and the refractive index of polyimide derived from Cavadi et al. 2001[20]. Upon our experience, based on UV/Vis transmission measurements[21] and X-ray photoelectron spectroscopy measurements[9], we have assumed that an oxide layer of $50 \AA$ builds up on each side of the aluminum coating on polyimide and becomes nearly transparent to UV/Vis radiation. For this reason, only the effective bulk aluminum layer has been conservatively taken into account in the calculation.

In order to cover the full field of view of the WFI, the OBF, located nearly $10 \mathrm{~cm}$ above the detector plane, will be significantly larger than the WFI sensitive area, either square $160 \times 160 \mathrm{~mm}^{2}$ or circular with $225 \mathrm{~mm}$ diameter (Figure 4). The choice between circular or square shape is discussed in a next paragraph based on structural analysis. A small detector (64x64 pixels) with the same DEPFET technology but faster read-out ( $80 \mu$ s time resolution vs. $1.3 \mathrm{~ms}$ of the imaging detector) is located on the side of the main detector (orange square in Figure 4). The design of the optical blocking filter for the fast detector is not very challenging and is not discussed in this paper.

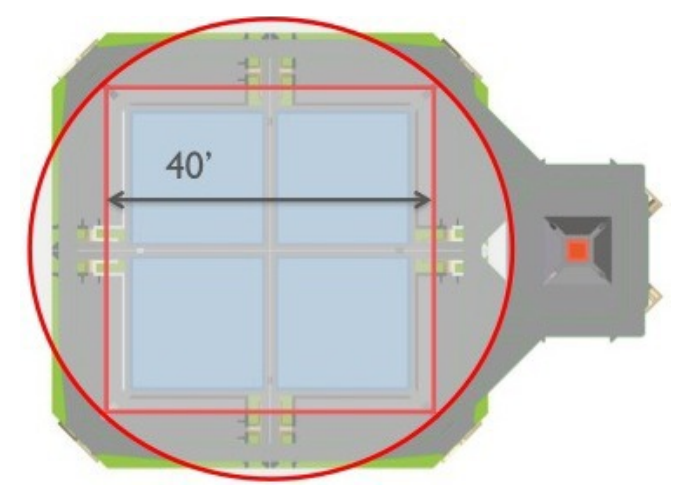

Figure 4. Layout of the square and circular filters with proper sizes to allow full field illumination of the WFI consisting of four separate detector quadrants each with $512 \times 512$ pixels.

\section{OPTICAL LOAD FROM ASTROPHYSICAL SOURCES}

Nearly every UV/Vis photon reaching the sensitive layer of the detector produces an electron-hole pair which adds to the $\mathrm{X}$-ray photon induced pairs deteriorating the energy resolution and shifting the energy scale by $3.7 \mathrm{eV}$ per pair on average. Ideally, the OBF (on-chip + FW) should provide a UV/Vis attenuation level such that less than one electronhole pair is produced by UV/Vis photons during the integration time of an X-ray photon.

In the following we have calculated the number of electron-hole pairs that are generated by UV/Vis photons when observing massive stars, which among the known astrophysical sources have the largest values of the ratio $f_{\mathrm{uv}-v i s} / f_{\mathrm{x}}$. For these calculations we have used the synthetic spectra of the Ostar2002[22] and Bstar2006[23] grids. These grids were generated with the non-LTE plane-parallel model atmosphere code TLUSTY. These files provide the Eddington fluxes at the stellar surface as a function of frequency from the EUV into the far IR. These Eddington fluxes were converted into fluxes at Earth for a given spectral type and a given apparent magnitude. Bolometric corrections, needed for this conversion, were taken from Martins et al. (2005)[24] for the O-type stars and Lanz \& Hubeny (2007)[23] for the B-type stars. Only main-sequence stars were considered as the relevant fluxes depend mainly on effective temperature and apparent magnitude and are not very sensitive to the surface gravity.

The interstellar dust absorption was accounted for via the relation of Mathis (2000)[25]. We considered four values of the extinction $\mathrm{AV}=0.0,0.5,1.0$ and 1.5. This reddening law does not deal with the absorption by interstellar gas which is especially important at wavelengths shorter than $912 \AA$ where interstellar hydrogen blocks our view in most directions. We have thus assumed that no flux at wavelengths shorter than $912 \AA$ reaches the telescope. This assumption breaks 
down for a few early-type stars that are located in holes of the ISM. The ISM absorbed fluxes at Earth were integrated between 912 and $11060 \AA$, where the upper limit corresponds to the Si band gap. For the simulations, we further assumed an ATHENA telescope PSF HEW of 5" and effective area of $2 \mathrm{~m}^{2}$, a detector read-out time of $1.3 \mathrm{~ms}$ and an average energy per electron-hole pair of $3.7 \mathrm{eV}$.

Figure 5 shows, in the worst case scenario (no interstellar absorption), the calculated number of electron-hole pairs per pixel (2.23" x 2.23") generated by UV/Vis photons during the integration time of $1.3 \mathrm{~ms}$, as a function of the visual magnitude of the star and the $T_{\text {eff }}$ in the range $15000-45000 \mathrm{~K}$. The figure shows the results obtained considering only the attenuation of the on-chip OBF (continuous line), and the total attenuation with the addition of one of three different options for the FW OBF (dashed line), namely: $200 \mathrm{~nm}$ of polyimide and $40 \mathrm{~nm}$ of aluminum (left panel), $200 \mathrm{~nm}$ of polyimide and $30 \mathrm{~nm}$ of aluminum (central panel), and $150 \mathrm{~nm}$ of polyimide and $30 \mathrm{~nm}$ of aluminum (right panel).
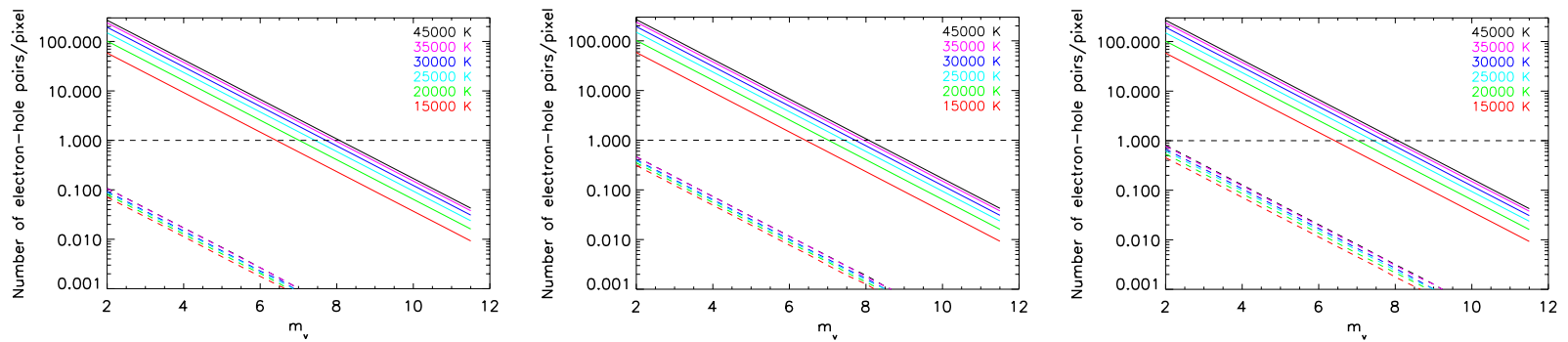

Figure 5: Predicted number of electron-hole pairs per pixel of the WFI generated by the observation of early-type stars without (solid lines) and with the addition of one of three different options for the FW OBF (dashed line), namely: $200 \mathrm{~nm}$ polyimide and $40 \mathrm{~nm}$ aluminum (left panel), $200 \mathrm{~nm}$ polyimide and $30 \mathrm{~nm}$ aluminum (central panel), and $150 \mathrm{~nm}$ polyimide and $30 \mathrm{~nm}$ aluminum (right panel).

Stars brighter than mv $\sim 7.5$ could not be observed only with the on-chip OBF. On the other hand, the capability to observe hot stars as bright as $\mathrm{mv}=2$ is mandatory to perform studies of X-ray variability to probe the dynamics of stellar winds in O-B stars[26]. Furthermore, stellar clusters that will likely be targets of the WFI contain early-type stars as bright as $\mathrm{mv}=4$. The use of an additional FW OBF is thus necessary.

All the three investigated FW OBF allow to observe even the brightest massive stars and thus in principle the thinnest one should be preferable to maximize X-ray transmission. With a similar approach we have calculated the number of electron-hole pairs per WFI pixel when observing the planet Mars, which is the brightest in the Solar system $\left(\mathrm{m}_{\mathrm{v}}=3.8\right.$ $4.7 \mathrm{mag} / \operatorname{arcsec}^{2}, \mathrm{~T}_{\mathrm{EFF}}=3500 \mathrm{~K}$ ). Using only the on-chip $\mathrm{OBF}$ we have 138 electron-hole pairs/pixel, while with the thickest OBF we obtain 0.75 electron-hole pairs/pixel during the WFI integration time of $1.3 \mathrm{~ms}$.

The choice of the proper FW OBF will be the outcome of an optimization analysis that will be performed in the near future based also on the results of the structural analysis as well as on the future progresses in telescope and detector design (e.g. improving the telescope PSF from the 5" HEW baseline to the 3" HEW goal determines an increase of nearly a factor 3 in the number of optically generated electron-hole pairs per pixel).

\section{FILTER STRUCTURAL ANALYSIS}

Given the very large size of the FW OBF, and knowing that the filter will have to withstand severe acoustic and vibration loads during launch, the use of a reinforcing structure to mechanically support the thin polyimide film coated with aluminum has been investigated. In this section we report preliminary results obtained by non-linear static analyses, carried out using the general purpose code Ansys Mechanical. The materials considered for the supporting structures (stainless steel or titanium alloy) have been considered indefinitely linear-elastic, since in the final design no plasticity shall be admitted. The outer frame has been presently assumed infinitely stiff. Finally, since the actual load levels the filter will have to withstand during launch (mechanical random vibration, acoustic loads, differential pressure) are not yet known, we have initially performed structural analysis under static uniform pressure up to a maximum value of 10 mbar which was the qualification level for the Newton-XMM EPIC filters. For this reason the present results have to be considered preliminary and meaningful only in terms of relative comparison between different configurations. That said, the main results of the performed structural analysis can be here summarized. 


\section{Square vs. Circular Filter Shape}

At first we compare the behaviour under static pressure of square $160 \times 160 \mathrm{~mm}^{2}$ filter vs. circular filter with $225 \mathrm{~mm}$ diameter, both fully covering the FOV of the WFI detector. All meshes considered in this first analysis are realised by stainless steel. Gravity (whose effects are nearly negligible) + static pressure from 0 to $10 \mathrm{mbar}$ has been applied on the reinforcing mesh. Figure 6 shows the results of this analysis in terms of central deflections and maximum stress peak in the mesh wires. The square filter performs relatively better than the circular filter. Based on these results and considering the advantage of the smaller area we have chosen the square shape as the baseline design.
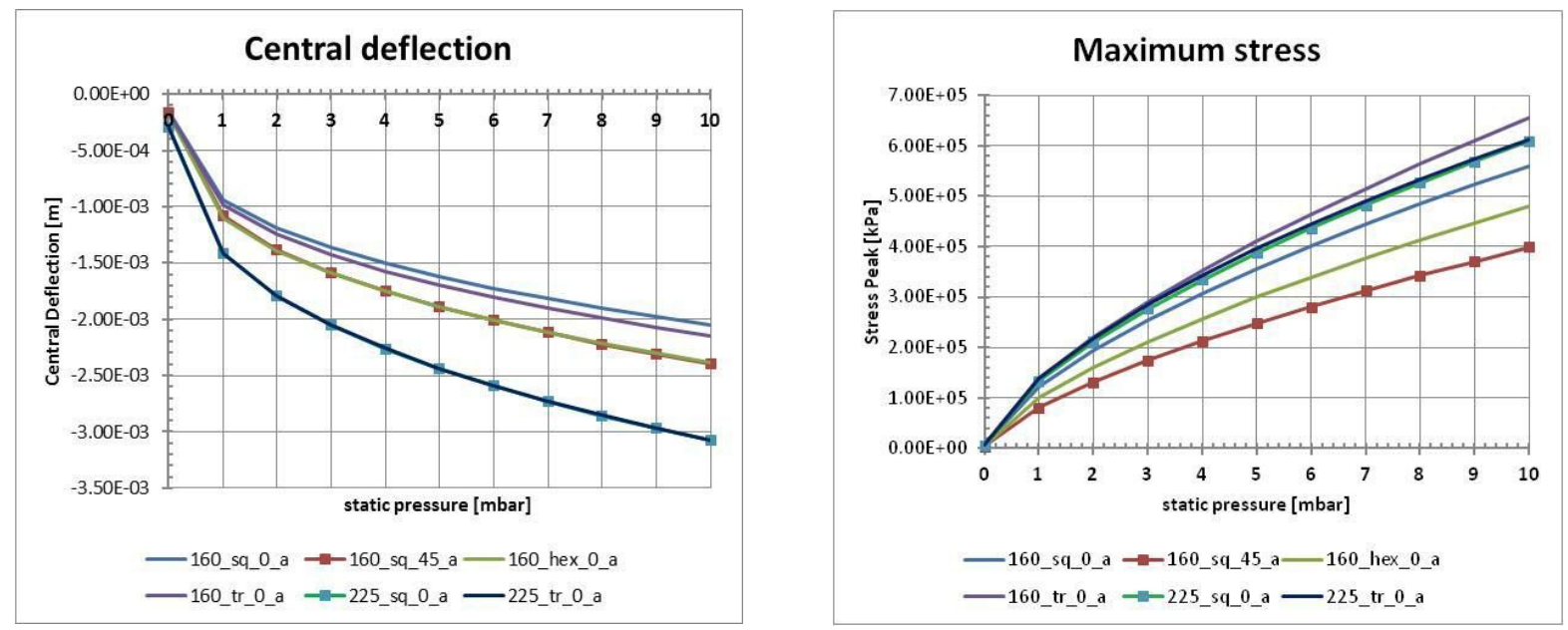

Figure 6: Left panel - maximum mesh deflection under static pressure ranging between zero (only gravity) and 10 mbar for different mesh patterns $(\mathrm{sq} 0=$ squares, $\mathrm{sq} 45=$ squares with wires parallel to square frame diagonals, hex $=$ hexagonal, $\operatorname{tr}=$ triangular) and frame shape (160 stands for square $160 \times 160 \mathrm{~mm}^{2}, 225$ stands for circular with 225 mm diameter). Right panel - maximum wire stress under static pressure.

\section{Mesh geometry and material}

Independently on the mesh pattern used (triangular, square or hexagonal), the same geometrical blocking factor is obtained, provided that the same wire width and the same distance between elementary cell centres is used. Under uniform pressure the wires in the central zone of the mesh are mainly subject to membrane tensile stresses. On the contrary at the mesh edges the maximum curvature occurs and the bending stresses prevail on membrane ones. Bending stresses at the edges are thus responsible of the maximum stress peaks in the mesh wires. Different mesh patterns have been investigated to support the thin filter mounted on the square frame (Figure 7), namely: square with wires parallel to frame walls, square with wires parallel to square fram diagonals, hexagonal, and triangular.
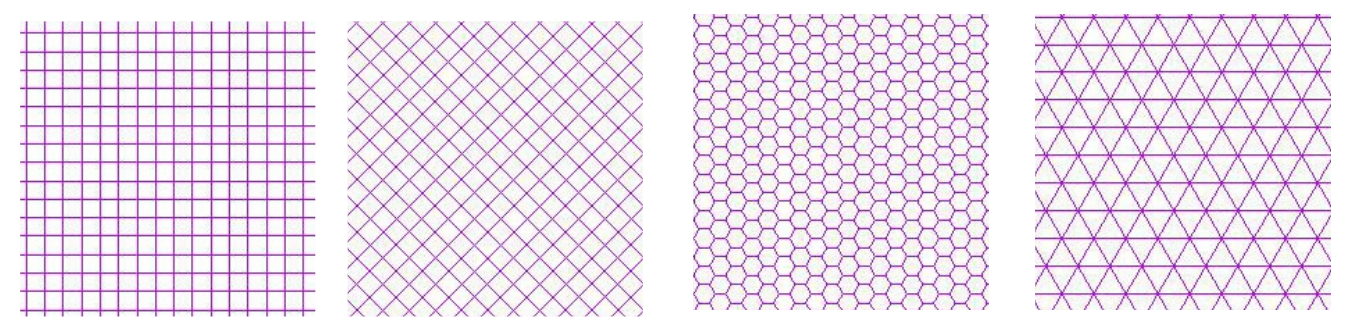

Figure 7: Mesh patterns investigated, namely: squares, squares with wires parallel to the square frame diagonals, hexagonal, triangular. 
Under static pressure, the square mesh pattern directed along filter diagonals (second from left in Figure 7), allows a more uniform load distribution, reducing the stress peaks at the filter edges (Figure 6 right panel). The hexagonal mesh pattern behaves second in the list, however, an optimization of the wires attachment onto the filter frame could make this pattern as good as the previous one. The stress peaks is larger than the tensile yield of stainless steel (530 MPa) for all the other investigated cases.

Various parametric analyses have been carried out to investigate the impact on stress peaks under uniform static pressure due to: wire depth, cell dimensions and wire width. A baseline mesh design is presented later in this paragraph, however, a more detailed optimization of the mesh design will be performed with structural analysis under dynamic load in a more advanced phase of the project development.

Most of the analyses have been carried out by considering stainless steel meshes. Nevertheless, it is possible to reduce the bending stress peaks at the edges by using a material with lower intrinsic stiffness (smaller Young modulus). Titanium alloys seem good candidates since they have smaller Young modulus with respect to stainless steel (approximately $115 \mathrm{GPa}$ vs. $210 \mathrm{GPa}$ ), high strength (tensile yield of $880 \mathrm{MPa}$ vs. $530 \mathrm{MPa}$ ), and relatively good fatigue strength. Moreover Ti alloy are lighter that stainless steel (density approximately $4.5 \mathrm{t} / \mathrm{m} 3 \mathrm{vs} .8 .0 \mathrm{t} / \mathrm{m} 3$ ) and so the stresses induced by mechanical vibrations will be smaller. The use of stainless steel meshes is also not desirable since it can cause an increased BKG due to proton induced fluorescence $\mathrm{Fe}$ line in a spectral region that is particulary interesting for different scientific targets of ATHENA.

\section{Frame design and inner cross stiffener}

As a baseline frame type we have considered the two parts type design by LUXEL corp. previously adopted for the Chandra HRC-I UV/Ion shield and modified to the proper size of the WFI filter (Figure 8). Two configurations with two or three screws on each side have been compared for the outer frame $\left(6.5 \times 3 \mathrm{~mm}^{2}\right.$ cross section). Some local stress concentration occurs near the screws i.e. in correspondence to the points where loads are transmitted to the filter wheel. The configuration with 12 screws is a little bit worse both in terms of stress peak and stress range in static load. Nevertheless, considering the very small difference in terms of stress ranges, we deem better the configuration with 12 screws, since it avoids long unsupported sides, which are risky in terms of vibrations.
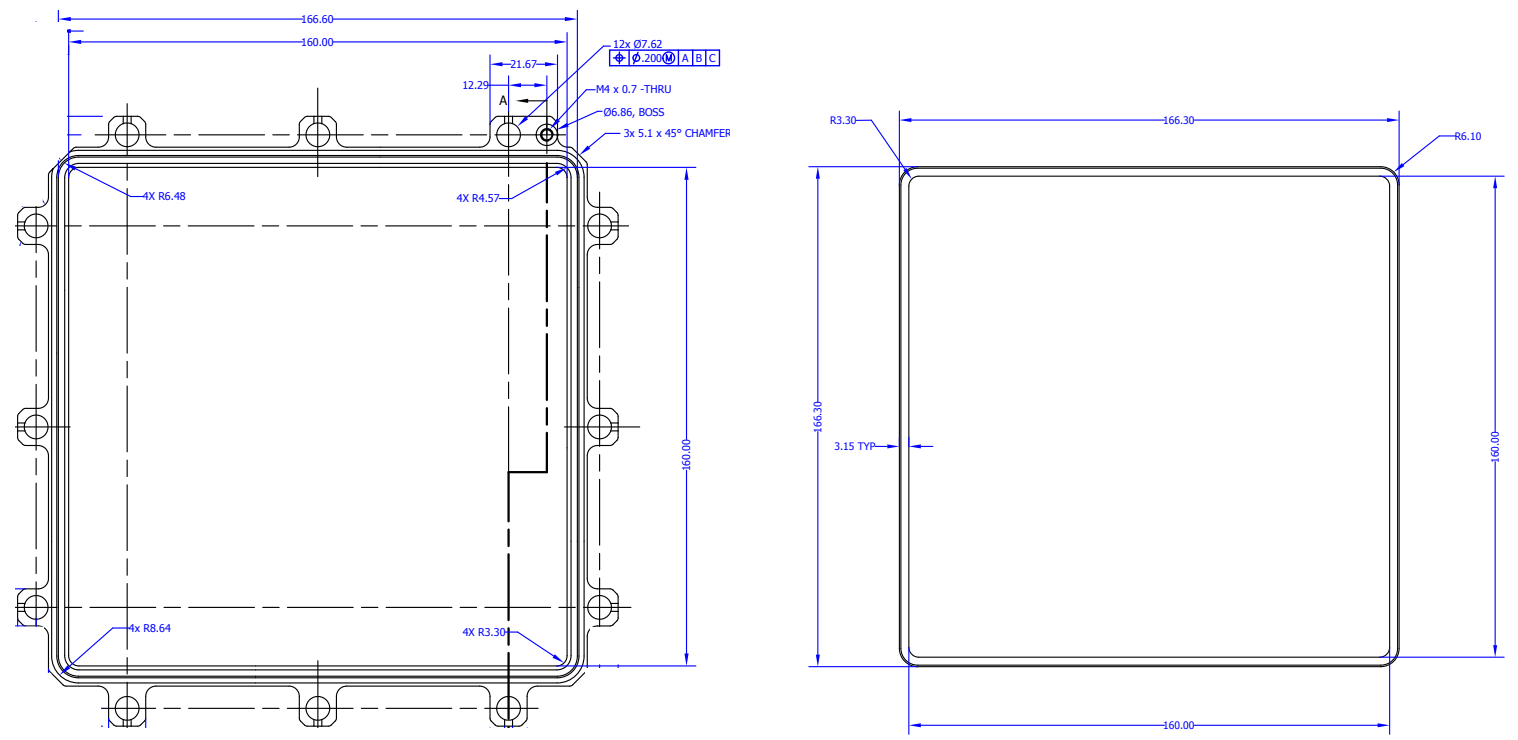

Figure 8: Current baseline WFI filter frame design based on the LUXEL corp. two parts type design previously used for the Chandra HRC-I UV-Ion shield. 
By introducing a stiffener dividing the filter in four equal quadrants (Figure 9 left panel) it is possible to reduce the mesh stress peak and deformation, under uniform static pressure, to the same level existing in a filter $80 \times 80 \mathrm{~mm}^{2}$. Nevertheless, in dynamic conditions this configuration could be potentially dangerous. The risk being related to the vibrations of the inner stiffeners which are free on the whole length. The use of the cross shaped stiffener has a second drawback, in fact, despite it matches the shape of the gap between the four WFI quadrants (see figure 4), it partially shades the area around the gap of the detector. Figure 9 - right panel shows the results of a ray-tracing simulation of a diffuse source focused by the ATHENA telescope onto the WFI when a cross-shaped stiffener with $2 \mathrm{~mm}$ wide bars supports the filter located $10 \mathrm{~cm}$ above the focal plane. The yellow dashed lines define the area around the detector gap where the QE is reduced by more than 5\% due to the partial shadow of the stiffener. For these two reasons we do not forsee to use a cross shaped stiffener in addition to the mesh reinforcing structure.
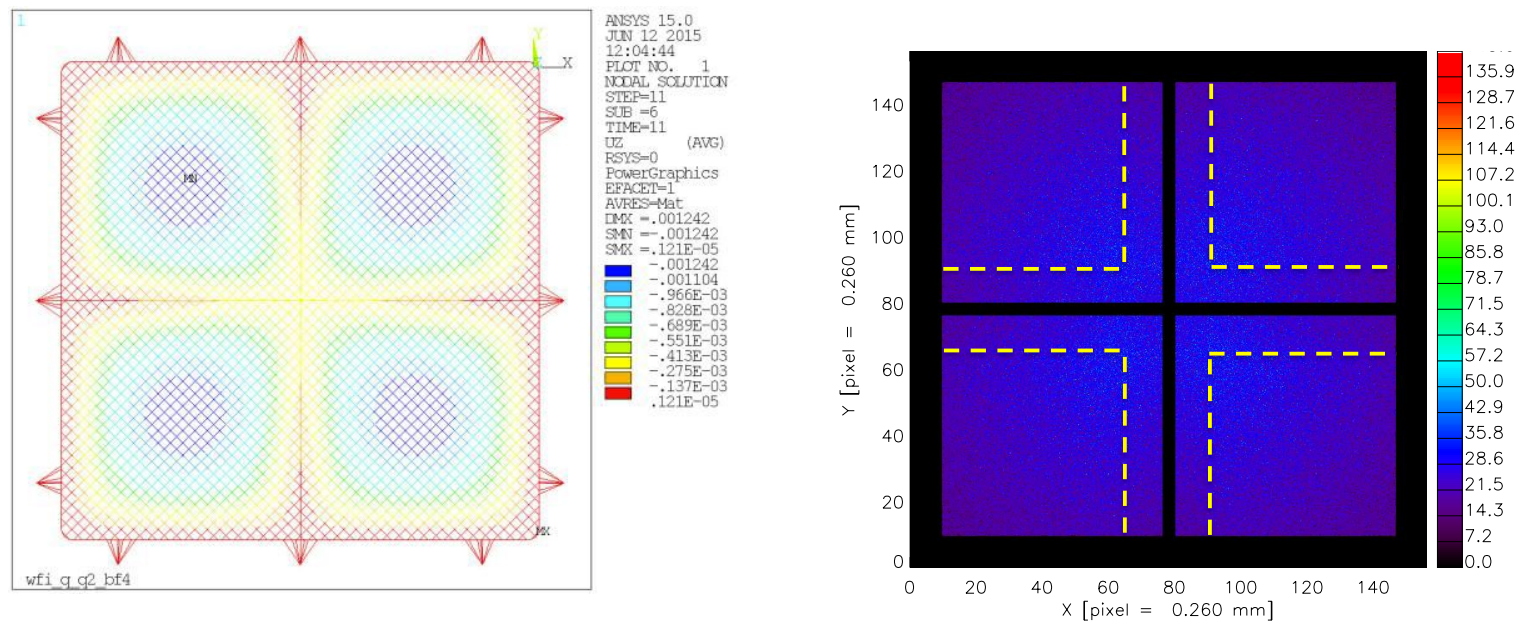

Figure 9: Left panel - Maximum mesh deformation under 10 mbar static pressure (units are meters) with a cross shaped stiffener with $2 \mathrm{~mm}$ wide $2 \mathrm{~mm}$ thick bars. Right panel - Ray-tracing simulation of a diffuse source. The yellow dashed lines define the area around the detector gap where the QE is reduced by more than 5\% due to the partial shadow of the cross haped stiffener.

\section{Stress in the polyimide film}

In order to evaluate stresses in the polyimide film under 10 mbar uniform static pressure we have adopted a supporting mesh defined on the basis of the results of a preliminary trade-off analysis. The main data of the mesh are: square geometry with elementary cell size $=2.923 \mathrm{~mm}$ and wires parallel to the square frame diagonals, wire width $=70 \mu \mathrm{m}$, wire thickness $=250 \mu \mathrm{m}$, mesh material Ti alloy Ti6Al4V, resulting optical blocking factor $=4.8 \%$. For the polyimide film thickness we have adopted $150 \mathrm{~nm}$ which is the smallest value considered up to now on the basis of the optical load analysis. To speed up the CPU time, just one quarter of the system composed by mesh+film is modelled, using symmetry constraints along boundaries through the filter centre. Figure 10 shows the maximum deflection and maximum membrane stress on the polyimide film. The maximum resulting stress on the polyimide film is $46.6 \mathrm{Mpa}$ well below the ultimate tensile stress of $310 \mathrm{MPa}^{1}$. The maximum stress peaks in the mesh wires, located at the outer edges, are equal to $600 \mathrm{MPa}$ which are quite high but still with a residual margin with respect to the nominal tensile yield value of Ti alloy Ti6Al4V (880 MPa).

\footnotetext{
${ }^{1}$ Technical sheet for LUXfilm ${ }^{\circledR}$ polyimide by LUXEL corp.
} 

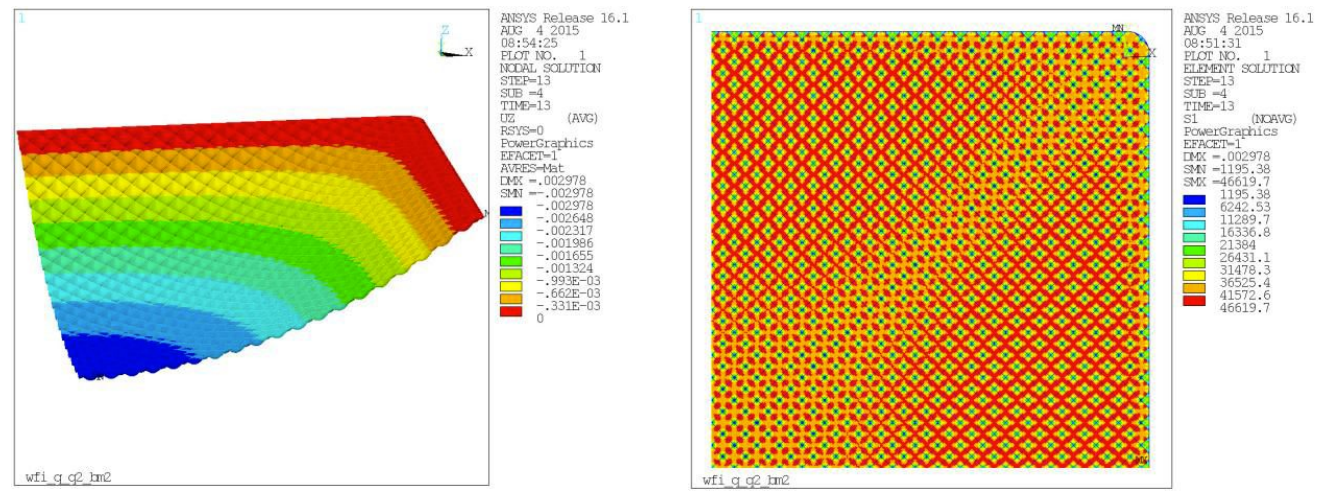

Figure 10: Left panel - Magnified maximum deformation (units are meters) under 10 mbar uniform static pressure of a polyimide film $150 \mathrm{~nm}$ thick supported by a Ti alloy mesh with $<5 \%$ blocking factor (maximum deformation $=2.98 \mathrm{~mm}$ ). Right panel - Maximum tensile stress (units are $\mathrm{KPa}$ ) of the film under the same conditions.

\section{FILTER WHEEL ASSEMBLY}

The Filter Wheel (FW) contains one filter, the calibration source, the closed aperture for background calibration and detector safety protection, and the open aperture for nominal operation. The filter wheel is rotated by stepper motor via gear. Commands to move the FW are sent by the Filter Wheel Controller while position of the filter wheel are checked by the position encoders. The diameter of the FW is currently approx $600 \mathrm{~mm}$. The whole module including the filter wheel, driving mechanics and electronics is named Filter Wheel Assembly (FWA). The technical challenges for the FWA mechanical structure, besides the proper functionality, are connected with the protection of the optical blocking filter. The FWA mechanical structure should include elements made of damping material (load absorber) or design solutions to minimize vibration and acoustic loads in the position where the filter will be located during launch.

The FWA structure will be loaded during launch by a combination of random vibration and acoustic excitation. The complexity in simulating such composite environment makes a full computer FEM simulation difficult. Since the acoustic loads are difficult to suppress and they can have a significant influence on the FWA and filter design, we have started our analysis considering only the acoustic loads. Here we present some preliminary results of the analysis aimed at verifying the effect of two possible design solutions to damp the acoustic load: 1) introducing a $5 \mathrm{~mm}$ thick plug at the entrance of the light baffle, 2) introducing a sound absorbing material close to the filter (Figure 11).

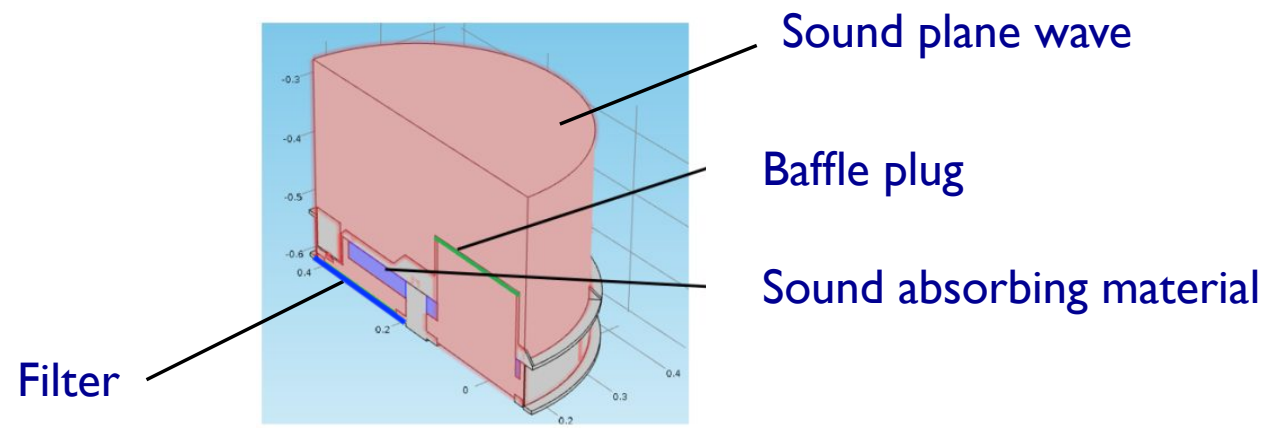

Figure 11: Schematic drawing of the FWA showing the filter and the two additional elements investigated to damp the acoustic load onto the filter: 1) the plug at the entrance of the light baffle, 2) the sound absorbing material close to the filter. The sound plane wave simulated to derive the sound pressure at the filter plane is also indicated.

The FEM modeling has been performed using Comsol Multiphysics. Initially, we have derived the eigenfrequencies of the structure with air inside, then we have applied a sound plane wave (see figure 11) for selected eigenfrequencies and have derived the sound pressure level on the filter plane for the three different cases: 1) no protection, 2) plug at the entrance of the baffle, and 3) plug plus sound absorbing material close to the filter. Figure 12 shows the pressure level at the filter plane for the three configurations. The sound pressure level on the filter is different for each eigenfreqency as a result of unique space distribution of sound pressure inside the FWA. The worst case is for $110 \mathrm{~Hz}$ and $770-836 \mathrm{~Hz}$. 


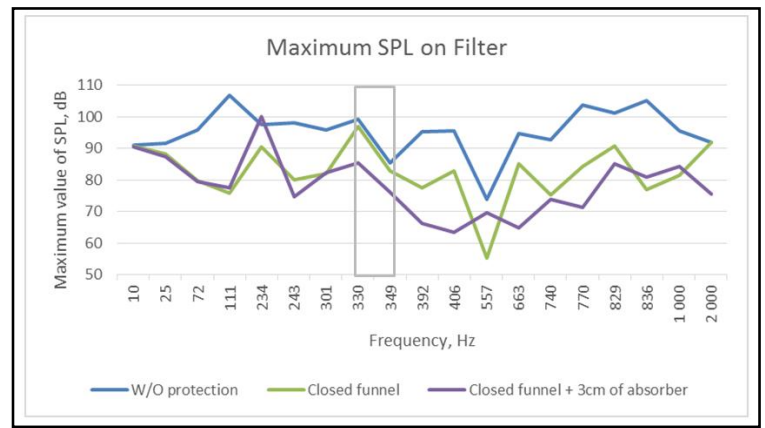

Figure 12: pressure level at the filter plane for the three investigated configurations: 1) no protection (blue), 2) plug at the entrance of the baffle (green), and 3) plug plus sound absorbing material close to the filter (violet).

Closing the baffle with a $5 \mathrm{~mm}$ thick aluminum plug reduces the maximum sound pressure level for most of the frequencies including the most dangerous ranges. However, the FWA with closed baffle has different eigenfrequencies, therefore, for some sound waves closing the baffle did not give any improvement ( 330 and $349 \mathrm{~Hz}$ ). Much better results could be obtained adding a sound absorption material inside the FWA. It reduces resonances inside and further reduces sound pressure level affecting the filter. Figure 13 shows the sound pressure level at the location of the filter in open position and in presence of one of the investigated damping solutions. In particular, damping effect with plug at $71 \mathrm{~Hz}$, and damping effect with plug + absorbing material at $330 \mathrm{~Hz}$ where the only plug would not be effective.

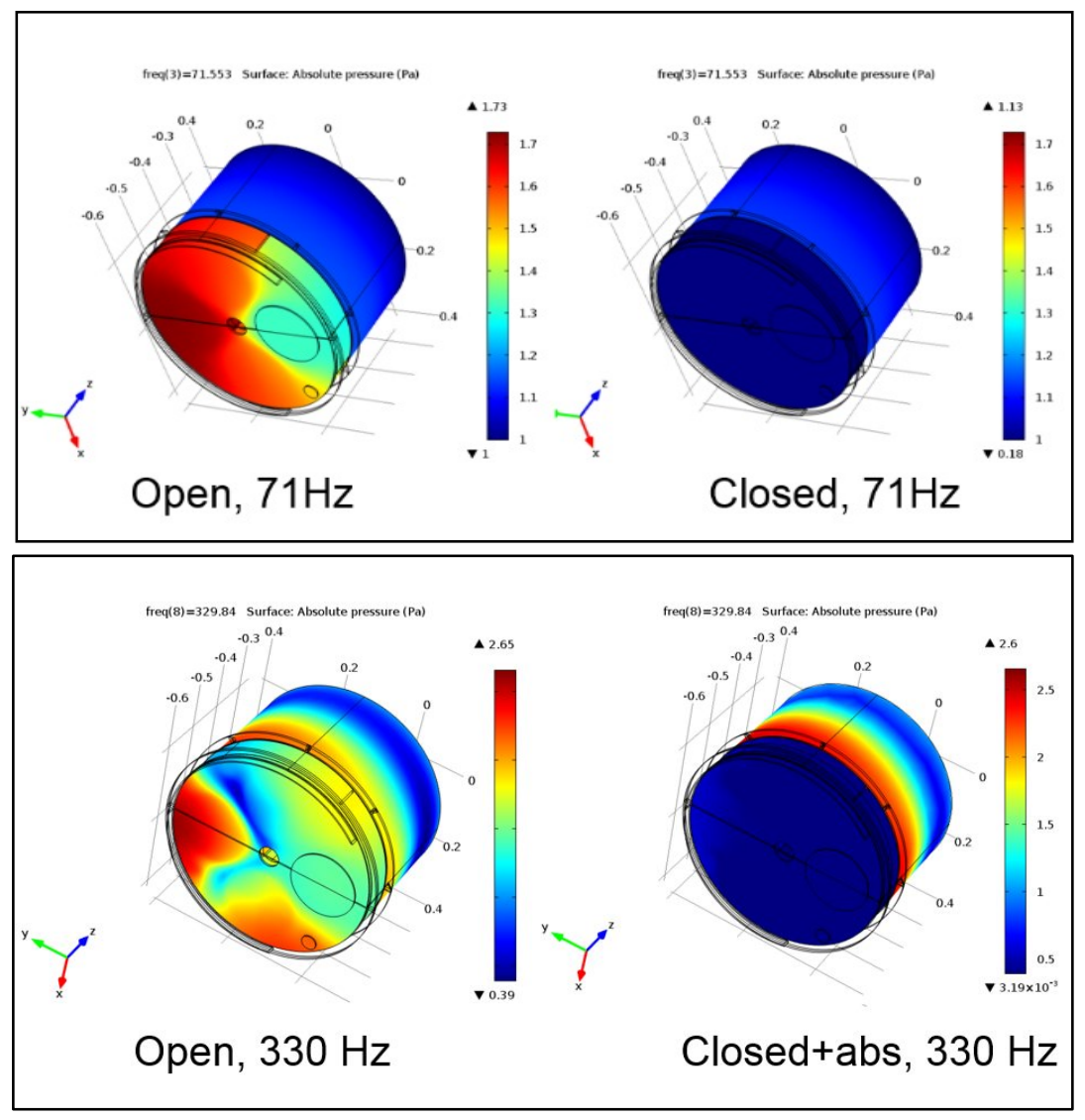

Figure 13: Sound pressure level at the filter plane (circle in the filter wheel) for two different frequencies and different damping solutions, namely plug at the entrance of the baffle $(71 \mathrm{~Hz})$, and plug plus sound absorbing material close to the filter $(330 \mathrm{~Hz})$. 
The present results of FEM analysis are mainly meningful in terms of relative comparison between different configurations and provide useful indications towards an efficient design of the FWA. Along the project development new simulations will be performed including a realistic sound pressure level representative of the environment in the launcher cargo and also including the vibrational load. Given the very complex system, test measurements will be needed to verify the simulations with a mock-up of the FWA and filter, in a representative vibro-acoustic environment.

\section{SUMMARY AND CONCLUSIONS}

The WFI detector is sensitive to UV/Vis photons with energy above the Si gap (1.12 eV). Optically generated electronhole pairs may degrade the spectral resolution as well as change the energy scale by introducing a signal offset. For this reasons, the use of an X-ray transparent optical blocking filter (OBF) is needed to allow the observation of X-ray sources with bright UV/Vis counterparts such as massive stars. The on-chip OBF consisting of $30 \mathrm{~nm} \mathrm{SiO}{ }_{2}-20 \mathrm{~nm} \mathrm{Si}_{3} \mathrm{~N}_{4}-90$ $\mathrm{nm} \mathrm{Al}$ provides nearly $10^{-5}$ attenuation at wavelengths $>1000 \AA$, however, the attenuation is nearly two orders of magnitude lower at shorter wavelengths where the BB emission of massive stars peaks. An additional OBF mounted on the filter wheel is thus needed.

Based on the successful heritage from previous missions using large area optical blocking filters, we have selected polyimide coated with aluminum as the baseline material for the construction of the WFI filter wheel OBF. Polyimide, beside its very good mechanical properties, is very opaque in the UV thus providing the needed additional attenuation.

Using synthetic spectra of massive stars, assuming an ATHENA telescope with a PSF HEW of 5" and effective area of $2 \mathrm{~m}^{2}$, a detector integration time of $1.3 \mathrm{~ms}$, and an average energy per electron-hole pair of $3.7 \mathrm{eV}$, we have estimated the number of optically generated electron-hole pairs detected by the WFI as a function of the stellar effective temperature and apparent visual magnitude. In particular, we have considered three different options for the FW OBF, namely: 200 $\mathrm{nm}$ of polyimide and $40 \mathrm{~nm}$ of aluminum, $200 \mathrm{~nm}$ of polyimide and $30 \mathrm{~nm}$ of aluminum, and $150 \mathrm{~nm}$ of polyimide and $30 \mathrm{~nm}$ of aluminum. Stars brighter than $\mathrm{mv} \sim 7.5$ cannot be observed with only the on-chip OBF. All three FW OBF investigated designs allow to observe even the brightest massive stars of interest.

Figure 14, shows the X-ray transmission of the on-chip optical blocking filter and the combination of the on-chip OBF and one of the three considered options for the FW OBF, all supported by a Ti mesh $250 \mu \mathrm{m}$ thick with $5 \%$ blocking factor.

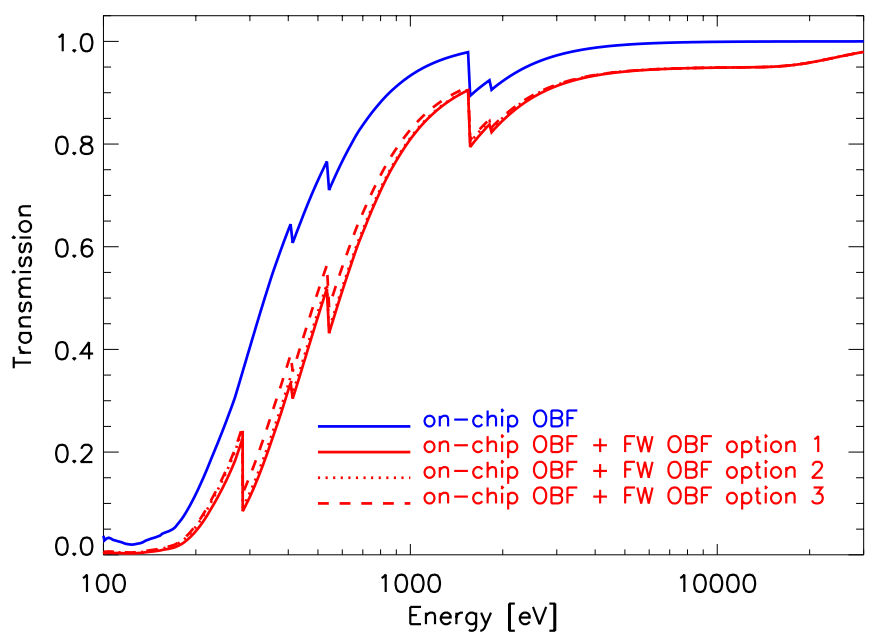

Figure 14. Calculated X-ray transmission of the multi-layer optical blocking filter directly deposited on the DEPFET consisting of $30 \mathrm{~nm} \mathrm{SiO}{ }_{2}-20 \mathrm{~nm} \mathrm{Si}_{3} \mathrm{~N}_{4}-90 \mathrm{~nm} \mathrm{Al}$ (blue line), and the total transmission including also one of three options for the FW OBF, namely: $200 \mathrm{~nm}$ of polyimide and $40 \mathrm{~nm}$ of aluminum (solid red line), 200 $\mathrm{nm}$ of polyimide and $30 \mathrm{~nm}$ of aluminum (dotted red line), and $150 \mathrm{~nm}$ of polyimide and $30 \mathrm{~nm}$ of aluminum (dashed red line). All the FW OBF include a Ti mesh $250 \mu \mathrm{m}$ thick with $5 \%$ blocking factor. 
The optical blocking filter is quite thin and with a very large size $\left(160 \times 160 \mathrm{~mm}^{2}\right)$, furthermore, the filter wheel will be launched in atmospheric pressure and thus the filter will have to withstand severe acoustic loads during the launch. For these reasons, the use of a reinforcing structure to mechanically support the thin polyimide film coated with aluminum has been investigated with FEM structural analysis. Only uniform static pressure has been considered up to now.

The use of a metal mesh made of a Ti alloy with a blocking factor lower than 5\% with square elementary cells and wires parallel to the square frame diagonals is efficient in terms of minimum deformation and wire stress. The maximum resulting stress on a polyimide film $150 \mathrm{~nm}$ thick is $46.6 \mathrm{Mpa}$ well below the ultimate tensile stress of $310 \mathrm{MPa}$. The maximum Stress peaks in the mesh wires are equal to $600 \mathrm{MPa}$ which is quite high but still with a residual margin with respect to the nominal yielding value $(880 \mathrm{MPa})$. The filter frame design is currently based on the LUXEL corp. two parts type design adopted for the Chandra HRC-I UV/Ion shield with three screws on each side.

In launch condition the filters are subject to quasi static accelerations, to mechanical vibration loads, and to acoustic loads since no vacuum launch is presently envisaged. Although we have currently simulated only static loads onto the mesh and filter, we have to consider that the 10 mbar static pressure on the square filter $160 \times 160 \mathrm{~mm}^{2}$ is equal to a force of $25.6 \mathrm{~N}$ which is more than 1000 times larger than the weight of the mesh $=0.0245 \mathrm{~N}$ (the weight of the film is negligible). This indicates that the tested level of static pressure is sufficiently high with respect to realistic levels of the mechanical vibrations $(<100 \mathrm{~g}$ RMS). On the contrary, more severe risks could arise from acoustic loads, from differential pressure during launch and from the combination of simultaneous loads. For these reasons it is necessary to have a simple preliminary evaluation (also in terms of static equivalent load) of all expected loads and of the combination criteria between simultaneous loads.

A critical item for the proper operation of the WFI is the filter wheel assembly which, besides its proper electromechanical functionality, has to provide protection to the large area optical blocking filter during launch. By use of FEM analysis a preliminary investigation has been performed to evaluate different options in the design of the FWA mechanical structure to minimize acoustic loads in the position where the filter will be located during launch.

The ongoing activities performed on the design of the OBF and FWA should bring to the definition of the conceptual design by the end of 2015. A first set of filter samples will then be procured to start preliminary vibro-acoustic tests in rapresentative environment to verify results of the FEM structural analysis and optimize the design of the filter and filter wheel assembly.

\section{ACKNOWLEDGEMENTS}

We acknowledge partial support by the Italian Space Agency (Contract number 2014-045-R.O.). We acknowledge fruitful discussions and suggestions by Bruce Lairson and Travis Ayers of LUXEL corp.

\section{REFERENCES}

[1] Barcons, X., Nandra, K., Barret, D., den Herder, J.-W., Fabian, A. C., Piro, L., Watson, M. G., and the Athena team, "Athena: the X-ray observatory to study the hot and energetic Universe", Journal of Physics Conference Series, Volume 610, Issue 1, article id. 012008 (2015).

[2] Nandra, K., Barret, D., Barcons, X., Fabian, A., den Herder, J.-W., Piro, L., Watson, M., Adami, C., Aird, J., Afonso, J. M., and et al., "The Hot and Energetic Universe: A White Paper presenting the science theme motivating the Athena+ mission", eprint arXiv:1306.2307N (2013).

[3] Willingale, R., Pareschi, G., Christensen, F., and den Herder, J.-W., "The Hot and Energetic Universe: The Optical Design of the Athena+ Mirror", eprint arXiv:1307.1709W (2013).

[4] Rau, A., Meidinger, N., Nandra, K., Porro, M., Barret, D., Santangelo, A., Schmid, C., Struder, L., Tenzer, C., Wilms, J., Amoros, C., Andritschke, R., Aschauer, F., Bahr, A., Gunther, B., Furmetz, M., Ott, B., Perinati, E., Rambaud, D., Reiffers, J., Treis, J., von Kienlin, A., Weidenspointner, G., "The Hot and Energetic Universe: The Wide Field Imager (WFI) for Athena+", eprint arXiv:1308.6785 (2013). 
[5] Meidinger, N., Nandra, K., Plattner, M., Porro, M., Rau, A., Santangelo, A. E., Tenzer, C., Wilms, J., "The wide field imager instrument for Athena", Proc. SPIE, 9144, id. 91442J 12 pp. (2014).

[6] Barbera M, Austin K, Collura A, Flanagan A, Jelinsky R, Murray S, Serio S, Zombeck M, "Development of the UV/ion shields for the Advanced X-ray Astrophysics Facility high-resolution camera (AXAF HRC)", Proc. SPIE, 2280, 214-228 (1994).

[7] Meehan R, Murray S, Zombeck V, Kraft P, Kobayashi K, Chappell H, Kenter T, Barbera M, Collura A, Serio S, "Calibration of the UV/ion shields for the AXAF High-Resolution Camera", Proc. SPIE, 3114, 74-100 (1997).

[8] Villa GE, Barbera M, Collura A, La Palombara N, Musso C, Serio S, Stillwell R, Tognon P, Turner DC The optical/UV filters for the EPIC experiment. IEEE Transactions on Nuclear Science, 45, 921-926 (1998).

[9] Barbera M, Agnello S, Buscarino G, Collura A, Gastaldello F, La Palombara N, Lo Cicero U, Tiengo A, Sciortino L, Varisco S, Venezia A M, "Status of the EPIC thin and medium filters on-board XMM-Newton after more than 10 years of operation: 1) laboratory measurements on back-up filters", Proc. SPIE, 8859, 88591412 pp. (2013).

[10] Gastaldello F, Barbera M, Collura A, La Palombara N, Lo Cicero U, Sartore N, Tiengo A, Varisco S, "Status of the EPIC thin and medium filters on-board XMM-Newton after more than 10 years of operation: 2) analysis of in-flight data", Proc. SPIE, 8859, 8859156 pp. (2013).

[11]Zane, S., et al., "The large area detector of LOFT: the large observatory for x-ray timing", Proc. SPIE, 9144, id. 91442W 19 pp. (2014).

[12]Feroci et al., "The Large Observatory for X-ray Timing (LOFT)", Experimental Astronomy, 34, issue 2, 415 (2012).

[13] Feroci M., et al., “The Large Observatory for x-ray timing”, Proc. SPIE, 9144, $91442 T 20$ pp. (2014).

[14] Barbera, M., Winter, B., Coker, J., Feroci, M., Kennedy, T., Walton, D., Zane, S., "Baseline design of the filters for the LAD detector on board LOFT", Proc. SPIE, 9144, 91446611 pp. (2014).

[15] Barbera M, Austin K, Collura A, Flanagan A, Jelinsky R, Murray S, Serio S, Zombeck M, "Development of the UV/ion shields for the Advanced X-ray Astrophysics Facility high-resolution camera (AXAF HRC)", Proc. SPIE, 2280, 214-228 (1994).

[16]Born M. and Wolf E., "Principles of Optics", 6th edition (1997), Cambridge University Press (UK), ISBN 0521639212.

[17] D.Y. Smith, "The optical properties of metallic aluminum", in Handbook of Optical Constants of Solids, p. 369-406 (1985), Edited by Edward D. Palik, ISBN: 978-0-12-544415-6

[18] Aleksandar D. Rakic., "Algorithm for the determination of intrinsic optical constants of metal films: application to aluminum", Appl. Opt. 34, 4755-4767 (1995).

[19] H.-J. Hagemann et al., "Optical constants from the far infrared to the x-ray region: $\mathrm{Mg}, \mathrm{Al}, \mathrm{Cu}, \mathrm{Ag}, \mathrm{Au}, \mathrm{Bi}, \mathrm{C}$, and Al2O3", J. Opt. Soc. Am. 65, 742-744 (1975).

[20] Cavadi A, Artale A, Barbera M, Collura A, Powell R, Varisco S, "Measurement of optical constants n and k of lexan and polyimide", Proc. SPIE, 3765, 805-815 (1999).

[21] Barbera M, Collura A, Artale A, Varisco S, Peres G, Sciortino S, Serio S, Villa G E., "Monitoring the stability of thin and medium back-up filters of the Newton-XMM EPIC camera", Proc. SPIE, 4851, 264-269 (2003).

[22] Lanz, T., \& Hubeny, I., "A Grid of Non-LTE Line-blanketed Model Atmospheres of O-Type Stars”, ApJS 146, 417 (2003).

[23]Lanz, T., \& Hubeny, I. , “A Grid of NLTE Line-blanketed Model Atmospheres of Early B-Type Stars”, ApJS 169, 83 (2007).

[24] Martins, F., Schaerer, D., \& Hillier, D.J., "A new calibration of stellar parameters of Galactic O stars “, A\&A 436, 1049 (2005).

[25] Mathis, J.S., in Allen's Astrophysical Quantities, 4th Edition, ed. A.N. Cox, AIP Press; Springer (New York), 523 (2000).

[26] Sciortino, S., Rauw, G., Audard, M., Argiroffi, C., Chu, Y.-H., De Becker, M., Drake, J., Feigelson, E., Gosset, E., Grosso, N., Güdel, M., Guerrero, M., Hervé, A., Kastner, J., Montez, R., Nazé, Y., Oskinova, L., Stelzer, B., ud-Doula, A., "The Hot and Energetic Universe: Star formation and evolution", eprint arXiv:1306.2333 (2013). 